\title{
Theranostic Nanoparticles
}

\author{
Feng Chen ${ }^{1}$, Emily B. Ehlerding ${ }^{2}$, and Weibo Cai ${ }^{1-4}$ \\ ${ }^{1}$ Department of Radiology, University of Wisconsin-Madison, Madison, Wisconsin; ${ }^{2}$ Department of Medical Physics, University of \\ Wisconsin-Madison, Madison, Wisconsin; ${ }^{3}$ Materials Science Program, University of Wisconsin-Madison, Madison, Wisconsin; and \\ ${ }^{4}$ University of Wisconsin Carbone Cancer Center, Madison, Wisconsin
}

\begin{abstract}
Theranostic nanoparticles hold the potential to revolutionize disease management. Over the last decade, there has been growing interest in the engineering of various kinds of theranostic nanoparticles for simultaneous cancer imaging and therapy in small animals. Efficient targeting of theranostic nanoparticles to the tumor site is critical for both diagnosis and therapy. However, difficulties still exist in the engineering of biocompatible theranostic nanoparticles with highly specific in vivo tumor-targeting capabilities. Here, we discuss the current and prospective status of theranostic nanoparticles that actively target tumors, as well as the challenges that still exist.
\end{abstract}

Key Words: theranostic nanoparticle; cancer; active targeting; theranostics; nanomedicine

J Nucl Med 2014; 55:1919-1922

DOI: 10.2967/jnumed.114.146019

Theranostic nanoparticles are multifunctional nanosystems well designed for specific and personalized disease management by virtue of their combining diagnostic and therapeutic capabilities into a single biocompatible, biodegradable particle (1). Ideal theranostic nanoparticles must accumulate rapidly and selectively in targets of interest, report biochemical and morphologic characteristics of disease, efficiently deliver sufficient drug on demand without damaging healthy organs, clear from the body within hours or be biodegraded into nontoxic byproducts, and be safe for humans (1). Although numerous types of theranostic nanoparticles, both organic and inorganic, have been developed over the last decade for treating cancer (Fig. 1) (2,3), none has yet satisfied all these criteria.

The value of active targeting of tumors has been demonstrated by many preclinical studies and clinical trials with peptide- or antibody-conjugated imaging nanoparticles and

Received Sep. 18, 2014; revision accepted Oct. 22, 2014.

For correspondence or reprints contact either of the following:

Feng Chen, Departments of Radiology and Medical Physics, University of

Wisconsin-Madison, Room 7137, 1111 Highland Ave., Madison, WI 53705-2275.

E-mail: fchen@uwhealth.org

Weibo Cai, Departments of Radiology and Medical Physics, University of

Wisconsin-Madison, Room 7137, 1111 Highland Ave., Madison, WI 53705-2275.

E-mail: wcai@uwhealth.org

Published online Nov. 20, 2014.

COPYRIGHT (c) 2014 by the Society of Nuclear Medicine and Molecular Imaging, Inc. chemotherapeutics $(4,5)$. Active targeting can also be particularly valuable in treating poorly vascularized small metastases $\left(<100 \mathrm{~mm}^{3}\right)$, for which enhanced permeability and retention alone may not be effective. So far, most previously reported uses of theranostic nanoparticles have focused on passive targeting strategies. It is still a major challenge to engineer biocompatible theranostic nanoparticles able to actively target tumors with high specificity. Here, we discuss the status, challenges, and future outlook for theranostic nanoparticles.

\section{GENERAL SYNTHETIC RULES AND TRANSLATIONAL RESEARCH}

Generally, theranostic nanoparticles can be engineered in several ways. For example, therapeutic agents (e.g., anticancer drugs and photosensitizers) may be conjugated, or loaded, to existing imaging nanoparticles such as quantum dots, iron oxide nanoparticles (IONPs), and gold nanocages. Another option is tagging of imaging contrast agents, such as fluorescent dyes, optical or magnetic nanoparticles, and various radioisotopes, to existing therapeutic nanoparticles. In addition, encapsulating both imaging and therapeutic agents together in biocompatible nanoplatforms such as polymeric nanoparticles, ferritin nanocages, and porous silica nanoparticles is also effective. Finally, engineering of unique nanoparticles (e.g., porphysomes, ${ }^{64} \mathrm{Cu}-\mathrm{CuS}$, and gold nanoshells or cages) with intrinsic imaging and therapeutic properties gives the desired results. For improving the blood circulation half-life and providing active tumor-targeting capability, surface modification with polyethylene glycol and different targeting ligands will usually be performed.

Although advancement of nanoparticle-based imaging and therapeutic agents into clinical trials is struggling because of toxicity concerns, progress has been made during the last decade. So far, the Food and Drug Administration has approved more than 35 imaging or therapeutic nanoparticles for clinical trials (6). Theranostic nanoparticles are still in the early translational stages, with nearly all efforts being devoted to preclinical studies and no clinical trials to date. The engineering of theranostic nanoparticles using Food and Drug Administration-approved imaging or therapeutic nanoplatforms may be a viable option. Platforms such as biodegradable polymeric nanoparticles, IONPs (currently used in clinical practice), gold nanoparticles or nanoshells (NCT00356980, NCT00848042), silica nanoparticles 


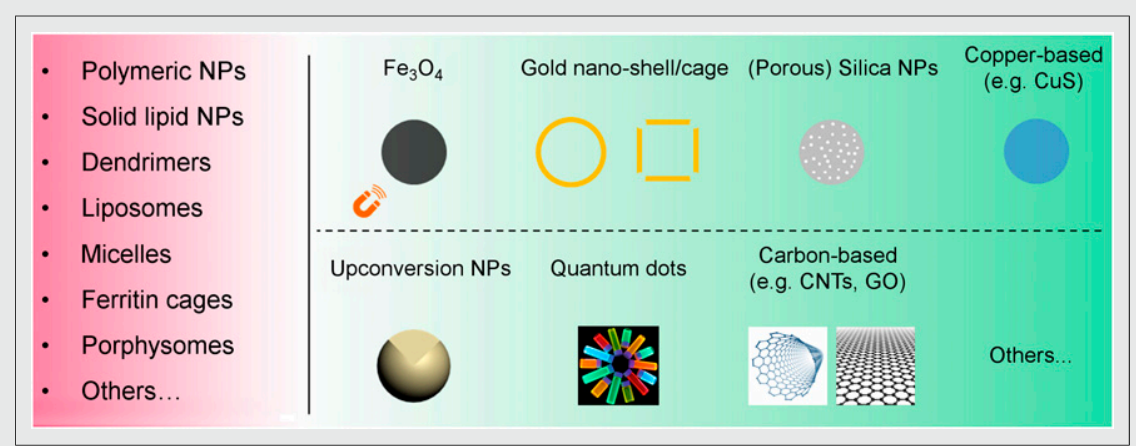

FIGURE 1. Organic and inorganic nanoplatforms for theranostic nanoparticle synthesis. CNTs = carbon nanotubes; GO = graphene oxide; NPs = nanoparticles. (Image of quantum dots was reprinted with permission of Evident Technologies.) overexpressed in many kinds of cancer, including ovarian and breast cancers (10). The porphysome nanostructure is a biodegradable, liposome-mimicking, intrinsic theranostic nanoparticle. Interestingly, the disassembly of this porphysome can convert the thermal ablation mechanism (i.e., photothermal therapy) back to the singlet oxygen $\left({ }^{1} \mathrm{O}_{2}\right)$ generation mechanism (i.e., photodynamic therapy). Inspired by this, researchers were able to develop targeting-triggered activatable nanobeacons by synthesizing folate-conjugated porphysomes (11). The results showed

(NCT02106598), and silica-gold nanoparticles (NCT01270139) may hold a greater chance of speeding up the translational process.

\section{ACTIVE TUMOR TARGETING AND RELATED LIGANDS}

Efficient targeting of theranostic nanoparticles to the tumor site is critical for both diagnosis and therapy. Some nanoparticles accumulate in tumor tissue on the basis of enhanced permeability and retention, whereby the leakiness of the tumor vasculature combined with poor lymphatic drainage enables accumulation within the tumor matrix (7). However, because of the tremendous heterogeneity in tumorvessel leakiness over space, time, and different types of tumors, as well as the unpredictable tumor extravasation of nanoparticles with varied sizes, shapes, and surface charges (8), the passive targeting strategy has its own limitations and may work only in certain fast-growing mouse and human xenograft tumors for nanoparticles with a relatively long blood circulation half-life.

In contrast, theranostic nanoparticles that actively target tumors are developed by further conjugating different targeting ligands to recognize and selectively bind to receptors that are overexpressed on certain tumor cell or tumor endothelial cell surfaces. Recently, tumor angiogenesis (or vasculature) targeting has been considered a generally applicable approach for most functionalized organic and inorganic nanomaterials (9). Depending on the physical and chemical properties of a specific theranostic nanoparticle, as well as the tumor model of interest, the targeting ligands may be antibodies, small peptides or molecules, lectins, aptamers, engineered proteins, or protein fragments. To date, only a few examples of theranostic nanoparticles that actively target tumor in vivo have been reported. Representative examples will be discussed in the following sections.

\section{TARGETING OF FOLATE RECEPTOR}

Folic acid is known to display high affinity (dissociation constant, $\sim 10^{-10} \mathrm{M}$ ) to the folate receptor, which is a glycosylphosphatidylinositol-linked membrane protein that is an efficient disruption of the porphysome nanostructure and the switching of photodynamic activity after the specific internalization of folate-conjugated porphysomes into tumor cells (Fig. 2). Other folate-conjugated theranostic nanoparticles include the heparin-folic acid-IR-780 nanoparticle (12) and folate-conjugated drug or dye-loaded IONPs (13).

\section{TARGETING OF INTEGRIN $\boldsymbol{\alpha}_{\mathrm{v}} \boldsymbol{\beta}_{3}$}

Peptide-modified ferritin nanocage (a protein-based nanoparticle made of 24 subunits, which can be selfassembled into a cagelike nanostructure) is another attractive biocompatible nanoplatform. It may be used for the targeted delivery of imaging agents (e.g., radiometal isotopes and fluorescent dyes) and therapeutic agents (e.g., photosensitizers and doxorubicin) to tumors that overexpress integrin $\alpha_{v} \beta_{3}$.

For example, nanocages loaded with $\mathrm{ZnF}_{16} \mathrm{Pc}$, which is modified with RGD4C were recently developed for in vivo integrin $\alpha_{\mathrm{v}} \beta_{3}$-targeted imaging and photodynamic therapy (14). Through replacement of $\mathrm{ZnF}_{16} \mathrm{Pc}$ with copperprecomplexed doxorubicin and near-infrared dye (ZW800),

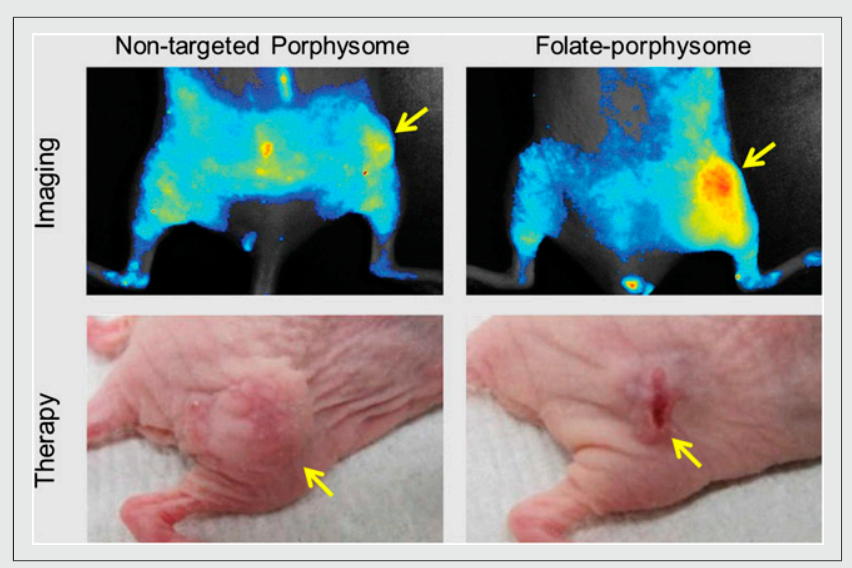

FIGURE 2. In vivo fluorescence activation imaging and photodynamic therapy response of nontargeted porphysome and folate-porphysome in KB tumor-bearing mice. Tumors are marked by arrows. a potent photosensitizer with a high ${ }^{1} \mathrm{O}_{2}$ quantum yield, and 
similar theranostic nanostructures have also been developed and have shown enhanced in vivo optical imaging and chemotherapeutic effects (Fig. 3) (15). RGD-modified $\mathrm{BaYbF}_{5}: \mathrm{Er}^{3+}$ nanocubes have also been reported for integrin $\alpha_{v} \beta_{3}$-targeted and CT image-guided radiotherapy. The PEGylated $\mathrm{BaYbF}_{5}: \mathrm{Er}^{3+}$ nanocubes were used as both the $\mathrm{CT}$ imaging contrast enhancer and the radiosensitizer in these studies (16). However, the potential long-term toxicity of the heavy metal contained in these nanocubes may hinder their future clinical translation.

\section{TARGETING OTHER CANCER MARKERS}

Besides folic acid and integrin $\alpha_{v} \beta_{3}$, well-designed theranostic nanoparticles have also been developed for targeting other receptors, such as prostate-specific membrane antigen (PSMA) in prostate cancer and the urokinase plasminogen activator receptor in pancreatic cancer $(17,18)$. For example, a theranostic nanoplex that contains multimodality imaging reporters (e.g., fluorescent dyes and radioisotopes) and a PSMA-targeting moiety was developed to deliver small interfering RNA and a prodrug enzyme to PSMA-expressing tumors (17). The nanoplex was carefully investigated using built-in noninvasive multimodality imaging to evaluate its diagnostic aspects in PSMA imaging, the therapeutic aspects of small interfering RNA-mediated downregulation of a target gene, and the conversion of prodrug to cytotoxic drug. No significant immune response or obvious toxicity to the liver or kidney was observed.

To overcome the physical barrier of tumor stroma, another study designed urokinase plasminogen activator receptortargeted IONPs, which also carry the chemotherapy drug

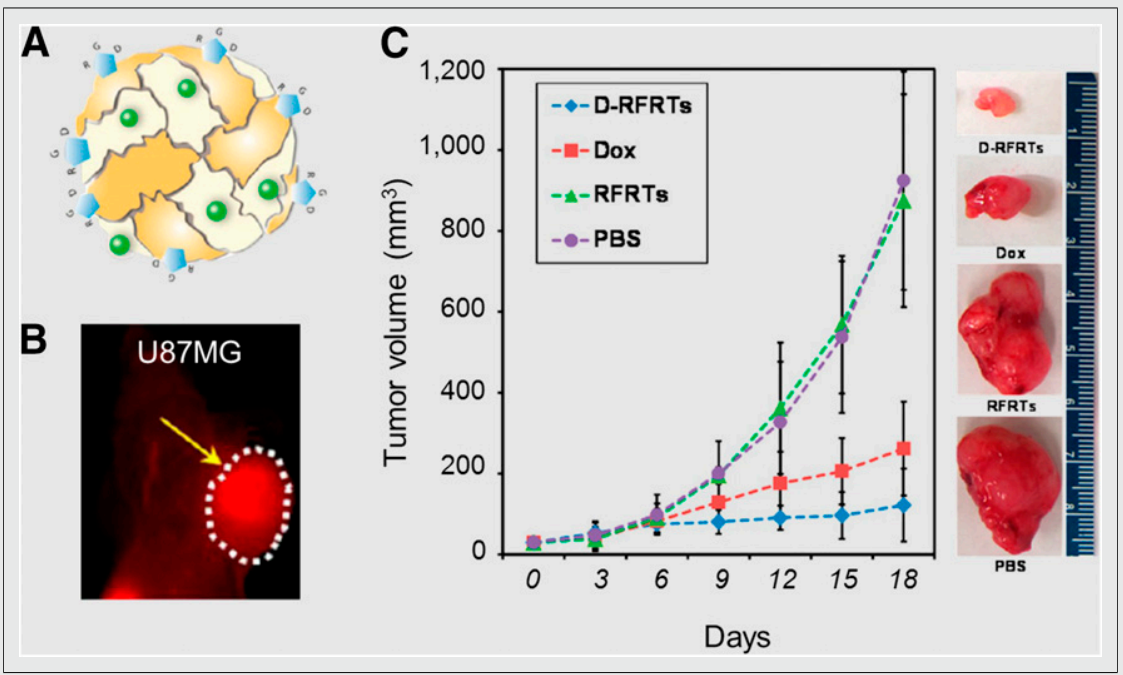

FIGURE 3. (A) Schematic illustration of copper-precomplexed doxorubicin and ZW800-coloaded ferritin nanocage. (B) In vivo targeted optical imaging of assynthesized ferritin nanocages in U87MG tumor-bearing mice. (C) In vivo chemotherapy study in U87MG tumor-bearing mice ( $n=5$ /group). Tumor is marked by arrow. Dox = doxorubicin; D-RFRTs = doxorubicin-loaded RFRTs; PBS = phosphate-buffered saline; RFRTs $=$ RGD4C-modified ferritins. gemcitabine, for targeted delivery into urokinase plasminogen activator receptor-expressing tumor and stromal cells (18). As synthesized, theranostic nanoparticles (i.e., ATFIONP-gemcitabine) enabled the intracellular release of gemcitabine after receptor-mediated endocytosis into tumor cells and provided contrast enhancement in MR imaging of tumors.

\section{CHALLENGES AND FUTURE DIRECTIONS}

Clearly, there is a trend toward combining the diagnostic and therapeutic functions of theranostic nanoparticles, resulting in greatly improved personalized disease management. For clinical translation to take place, many major challenges must be overcome, such as selection of the best nanoplatform, improvement of ligand conjugation efficiency, and development of an ideal synthetic technique with fewer steps, higher reproducibility, and lower cost. Because of the high sensitivity and accurate quantification of PET imaging, radiolabeling of the Food and Drug Administration-approved therapeutic nanoparticles, such as liposomal doxorubicin, could become a highly straightforward strategy to visualize and accurately assess the biodistribution, circulation halflife, and pharmacokinetics of theranostic nanoparticles, without compromising drug-loading capacity and safety. Conjugating targeting ligands to nanoparticles with intrinsic imaging and therapeutic properties, such as porphysomes and gold nanoshells and cages, will be another ideal way to develop future theranostic nanoparticles that actively target tumors.

Efforts at seeking better active-targeting strategies will continue. Recent research has shown that well-designed nanosystems can communicate in vivo to amplify cancertargeting efficacy (19). In a recent study, RGD-modified ferritin nanocages were synthesized as "smart" photosensitizer carriers that were able to sitespecifically deliver ${ }^{1} \mathrm{O}_{2}$ to the tumor endothelium for altering vasculature permeability. The results of that study also demonstrated that such external stimuli facilitated extravasation of the second dose of imaging or therapeutic nanoparticles at the tumor site (20). Although still in the early stages of development, such a concept as this in vivo cooperative targeting holds great potential for improving tumor-targeting capabilities and may become one of the most interesting research directions over the next 5 years.

There is also growing interest in developing activatable theranostic nanoparticles for even smarter cancer imaging and therapy. For example, an activatable theranostic prodrug was designed 
by conjugating $\mathrm{SN}-38$ (a topoisomerase inhibitor) with piperazine-rhodol fluorophore using a self-immolating linker based on disulfide bonds. Such a design may permit realtime monitoring of the delivery and release of the SN-38 payload in the presence of intracellular thiols (21). With activatable theranostic nanoparticles, getting vital information about treatment response may also be possible, which will be extremely helpful during the decisionmaking process and may, in time, help doctors alter treatment protocols.

\section{CONCLUSION}

The last decade has witnessed an unprecedented expansion in the engineering of various kinds of theranostic nanoparticles for cancer imaging and therapy (22-24). This short review has discussed the current and prospective status and challenges of active tumor targeting by theranostic nanoparticles. The development of theranostic nanoparticles that are tumor-specific, safe, simple, yet powerful—holding greater potential to be translated into the clinic-will continue to be the future focus.

\section{DISCLOSURE}

This work is supported in part by the University of Wisconsin-Madison, the National Institutes of Health (NIBIB/NCI 1R01CA169365, P30CA014520), the Department of Defense (W81XWH-11-1-0644), and the American Cancer Society (125246-RSG-13-099-01-CCE). No other potential conflict of interest relevant to this article was reported.

\section{REFERENCES}

1. Jokerst JV, Gambhir SS. Molecular imaging with theranostic nanoparticles. Acc Chem Res. 2011;44:1050-1060.

2. Xie J, Lee S, Chen X. Nanoparticle-based theranostic agents. Adv Drug Deliv Rev. 2010;62:1064-1079.

3. Lammers T, Aime S, Hennink WE, Storm G, Kiessling F. Theranostic nanomedicine. Acc Chem Res. 2011;44:1029-1038.

4. Blackwell KL, Burstein HJ, Storniolo AM, et al. Overall survival benefit with lapatinib in combination with trastuzumab for patients with human epidermal growth factor receptor 2-positive metastatic breast cancer: final results from the EGF104900 Study. J Clin Oncol. 2012;30:2585-2592.

5. Benezra M, Penate-Medina O, Zanzonico PB, et al. Multimodal silica nanoparticles are effective cancer-targeted probes in a model of human melanoma. J Clin Invest. 2011;121:2768-2780.

6. Thakor AS, Gambhir SS. Nanooncology: the future of cancer diagnosis and therapy. CA Cancer J Clin. 2013;63:395-418.

7. Fang J, Nakamura H, Maeda H. The EPR effect: unique features of tumor blood vessels for drug delivery, factors involved, and limitations and augmentation of the effect. Adv Drug Deliv Rev. 2011;63:136-151.

8. Smith BR, Kempen P, Bouley D, et al. Shape matters: intravital microscopy reveals surprising geometrical dependence for nanoparticles in tumor models of extravasation. Nano Lett. 2012;12:3369-3377.

9. Chen F, Cai W. Tumor vasculature targeting: a generally applicable approach for functionalized nanomaterials. Small. 2014;10:1887-1893.

10. Sudimack J, Lee RJ. Targeted drug delivery via the folate receptor. Adv Drug Deliv Rev. 2000;41:147-162.

11. Jin CS, Cui L, Wang F, Chen J, Zheng G. Targeting-triggered porphysome nanostructure disruption for activatable photodynamic therapy. Adv Healthc Mater. 2014;3:1240-1249.

12. Yue $\mathrm{C}$, Liu $\mathrm{P}, \mathrm{Zheng} \mathrm{M}$, et al. IR-780 dye loaded tumor targeting theranostic nanoparticles for NIR imaging and photothermal therapy. Biomaterials. 2013;34:6853-6861.

13. Santra S, Kaittanis C, Grimm J, Perez JM. Drug/dye-loaded, multifunctional iron oxide nanoparticles for combined targeted cancer therapy and dual optical/ magnetic resonance imaging. Small. 2009;5:1862-1868.

14. Zhen Z, Tang W, Guo C, et al. Ferritin nanocages to encapsulate and deliver photosensitizers for efficient photodynamic therapy against cancer. ACS Nano. 2013;7:6988-6996.

15. Zhen Z, Tang W, Chen H, et al. RGD-modified apoferritin nanoparticles for efficient drug delivery to tumors. ACS Nano. 2013;7:4830-4837.

16. Xing $H$, Zheng $X$, Ren $Q$, et al. Computed tomography imaging-guided radiotherapy by targeting upconversion nanocubes with significant imaging and radiosensitization enhancements. Sci Rep. 2013;3:1751.

17. Chen Z, Penet MF, Nimmagadda S, et al. PSMA-targeted theranostic nanoplex for prostate cancer therapy. ACS Nano. 2012;6:7752-7762.

18. Lee GY, Qian WP, Wang L, et al. Theranostic nanoparticles with controlled release of gemcitabine for targeted therapy and MRI of pancreatic cancer. ACS Nano. 2013;7:2078-2089.

19. von Maltzahn G, Park JH, Lin KY, et al. Nanoparticles that communicate in vivo to amplify tumour targeting. Nat Mater. 2011;10:545-552.

20. Zhen Z, Tang W, Chuang YJ, et al. Tumor vasculature targeted photodynamic therapy for enhanced delivery of nanoparticles. ACS Nano. 2014;8:6004-6013.

21. Bhuniya S, Maiti S, Kim EJ. An activatable theranostic for targeted cancer therapy and imaging. Angew Chem Int Ed Engl 2014;53:4469-4474.

22. Mura S, Couvreur P. Nanotheranostics for personalized medicine. Adv Drug Deliv Rev. 2012;64:1394-1416.

23. Muthu MS, Leong DT, Mei L, Feng S-S. Nanotheranostics: application and further development of nanomedicine strategies for advanced theranostics. Theranostics. 2014;4:660-677.

24. Puri A, Blumenthal R. Polymeric lipid assemblies as novel theranostic tools. Acc Chem Res. 2011;44:1071-1079. 